\title{
Association between intermittent low-back pain and superior cluneal nerve entrapment neuropathy
}

\author{
Yasuhiro Chiba, MD, PhD, ${ }^{1}$ Toyohiko Isu, MD, PhD, ${ }^{1}$ Kyongsong Kim, MD, PhD, ${ }^{2}$ \\ Naotaka Iwamoto, MD, PhD, ${ }^{1}$ Daijiro Morimoto, MD, PhD, ${ }^{3}$ Kazuyoshi Yamazaki, MD, ${ }^{1}$ \\ Masaaki Hokari, MD, PhD, ${ }^{1}$ Masanori Isobe, MD, PhD, ${ }^{1}$ and Mitsuo Kusano, MD, PhD ${ }^{4}$ \\ 'Department of Neurosurgery, Kushiro Rosai Hospital, Hokkaido; 'Department of Neurosurgery, Chiba Hokuso Hospital, \\ Nippon Medical School, Chiba; ${ }^{3}$ Department of Neurosurgery, Nippon Medical School, Tokyo; and ${ }^{4}$ Department of Surgery, \\ Kushiro Rosai Hospital, Hokkaido, Japan
}

OBJECTIVE Superior cluneal nerve (SCN) entrapment neuropathy (SCNEN) is a cause of low-back pain (LBP) that can be misdiagnosed as a lumbar spine disorder. The clinical features and etiology of LBP remain poorly understood. In this study, 5 patients with intermittent LBP due to SCNEN who had previously received conservative treatment underwent surgery. The findings are reported and the etiology of LBP is discussed to determine whether it is attributable to SCNEN. METHODS Intermittent LBP is defined as a clinical condition in which pain is induced by standing or walking but is absent at rest. Between April 2012 and March 2013, 5 patients in this study who had intermittent LBP due to SCNEN underwent surgery. The patients included 3 men and 2 women, with a mean age of 66 years. The affected side was unilateral in 2 patients and bilateral in 3 (total sites, 8). The interval from symptom onset to treatment averaged 51.4 months; the mean postoperative follow-up period was 17.6 months. The clinical outcomes were assessed using the numerical rating scale (NRS) for LBP, the Japanese Orthopaedic Association (JOA) scale, and the Roland-Morris Disability Questionnaire (RDQ) preoperatively and at the last follow-up; these data were analyzed statistically.

RESULTS None of the 5 patients reported LBP at rest. Intermittent LBP involving the iliac crest and buttocks was induced by standing or walking an average of $136 \mathrm{~m}$. In 2 patients with unilateral involvement, LBP was improved only by SCN block. Surgeries were performed on 6 sites in 5 patients because the SCN block was only transiently effective. Patients' SCNs penetrated the orifice of the thoracolumbar fascia. SCN kinking at the orifice was exacerbated at the lumbar-extension provocation posture, and radiating pain increased upon manual intraoperative compression of the SCN in this posture. After releasing the SCN surgically, disappearance of the pain was intraoperatively confirmed by manual compression of the SCN with the patients in the lumbar-extension posture. Surgery was effective in all 5 patients, and all clinical outcome scores indicated significant improvement $(p<0.05)$.

CONCLUSIONS To the authors' knowledge, this is the first report of patients with intermittent LBP due to SCNEN. Clinical and surgical evidence presented suggests that their LBP was exacerbated by lumbar extension and that symptom relief was obtained by SCN block or surgical release of the SCN entrapment. These results suggest that SCNEN should be considered as a causal factor in patients for whom walking elicits LBP.

http://thejns.org/doi/abs/10.3171/2015.1.SPINE14173

KEY WORDS superior cluneal nerve entrapment neuropathy; low-back pain; intermittent claudication

$\mathrm{L}$ ow-back pain (LBP) can be attributable to ligaments, facet joints, the vertebral periosteum, paravertebral musculature and fascia, and spinal nerve roots. Lumbar spinal stenosis (LSS) is a common cause of LBP. In patients with intermittent claudication, symptomatic improvement with spinal flexion, and evidence of spinal stenosis on imaging studies, LBP is considered to be due to $\mathrm{LSS}^{4-6}$
Superior cluneal nerve (SCN) entrapment neuropathy (SCNEN) is a cause of unilateral LBP involving the iliac crest and buttocks ${ }^{1-3,12-14,16,18,20}$ that tends to be misdiagnosed as lumbar spine disorder. The clinical features and etiology of LBP remain poorly understood..$^{13} \mathrm{LBP}$ due to SCNEN tends to be increased by lumbar extension. We report our surgical results in patients with intermittent LBP due to SCNEN and discuss its etiology.

ABBREVIATIONS JOA = Japanese Orthopaedic Association; LBP = low-back pain; LSS = lumbar spinal stenosis; NRS = numerical rating scale; RDQ = Roland-Morris Disability Questionnaire; SCN = superior cluneal nerve; SCNEN = SCN entrapment neuropathy; SIJ = sacroiliac joint.

SUBMITTED February 12, 2014. ACCEPTED January 16, 2015.

INCLUDE WHEN CITING Published online October 16, 2015; DOI: 10.3171/2015.1.SPINE14173. 


\section{Methods}

All patients gave prior written consent for participation in this study, which was approved by Kushiro Rosai Hospital's institutional review board. All patients had undergone unsuccessful conservative treatment, including perioral medication and SCN block, for intermittent LBP, defined as a motion-induced clinical condition in which pain is induced by standing or walking but is absent at rest. ${ }^{17}$

We excluded patients in whom pain relief was achieved by conservative treatment. None of the treated patients had undergone iliac crest harvest for grafting, suffered trauma to the affected area, or reported rapid weight gain. We also excluded patients with severe or suspected sacroiliac joint (SIJ) pain.

\section{Criteria for the Diagnosis of SCNEN}

In a previous study, ${ }^{14}$ we proposed criteria for the diagnosis of SCNEN. These include unilateral LBP involving the iliac crest and buttocks, a trigger point over the posterior iliac crest located $7 \mathrm{~cm}$ from the midline (corresponding to the nerve compression zone), and numbness and radiating pain in the SCN area upon compression of the trigger point (Fig. 1). Although symptom relief in more than $75 \%$ of patients was obtained within 2 hours of inducing local nerve block by injecting $2 \mathrm{ml}$ of $1 \%$ lidocaine at the trigger point in the buttocks, ${ }^{11,13,19}$ some patients reported pain recurrence after the analgesic effect of the test injection wore off. The SCN treated in our patients was located approximately $7-8 \mathrm{~cm}$ from the midline, and $\mathrm{SCN}$ pain is different from SIJ pain. However, to avoid mistaking pain from other sites as SCN pain, we injected a small amount of an analgesic locally. This eliminated anesthetization of sites such as the SIJ. In addition, the marked symptom improvement elicited by SCN block and the reproducibility of pain relief by repeat SCN block confirmed that patients in this study suffered from SCN pain.

Superior cluneal nerve entrapment neuropathy cannot

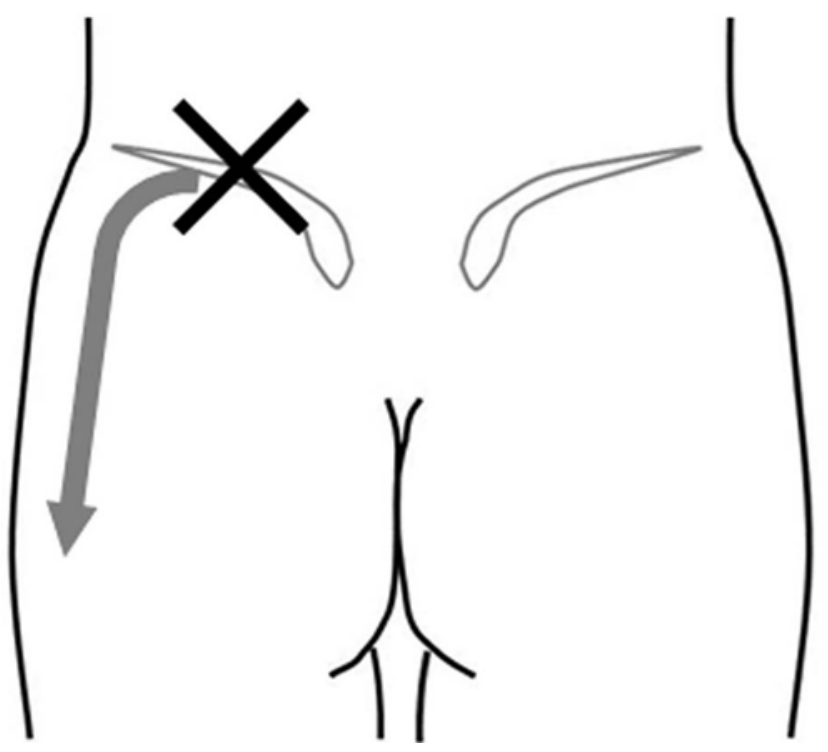

FIG. 1. Schematic illustration of the trigger point over the iliac crest $(X)$. Placing pressure on the trigger point induced radiating pain (arrow). be diagnosed by radiological or electromyographic studies. Also, various other conditions may produce symptoms similar to those of SCNEN. Based on radiological findings, we excluded patients with conditions that require special treatment, such as tumors and fractures. Before selecting our study subjects, we were careful not to attribute neurological symptoms solely to SCNEN.

\section{Patient Population}

Between April 2012 and March 2013, we treated 188 patients with SCNEN. Among them were 5 patients with intermittent LBP due to SCNEN. These were 3 men and 2 women ranging in age from 43 to 77 years (mean 66 years), who underwent intensive SCNEN treatment (Table 1). The affected side was unilateral in 2 patients and bilateral in 3 (total sites, 8). The duration of symptoms from onset to treatment averaged 51.4 months (range 3-156 months). We carefully assessed patients to ascertain that their LBP was attributable to SCN entrapment. The mean postoperative follow-up period was 17.6 months (range 15-22 months). Of the 5 patients, 4 were $>65$ years old; causal factors leading to the higher incidence of SCNEN among older compared with younger individuals remain to be identified. . $, 7,13,14,18^{2}$

\section{Surgical Technique}

As reported elsewhere, ${ }^{7,14}$ with the patient in the prone position and under local anesthesia, we performed microsurgical release of the SCN entrapment. We used only local skin anesthesia to avoid anesthetizing the SCN. We made a 5-cm-long skin incision across the trigger point, located $7 \mathrm{~cm}$ from the midline on the iliac crest. Then, we carefully dissected the subcutaneous soft tissue and identified the SCN with a nerve stimulator placed on the fat layer over the subcutaneous space. Indocyanine green video angiography helps to identify the SCN and its branches, and to confirm sufficient nerve decompression. ${ }^{7}$ When it was difficult to detect the SCN in the subcutaneous space, we could find it in the space under the thoracolumbar fascia. The elicitation of trigger point and traction pain is a good indication of the location of the SCN, which, together with its branches, slants from caudolateral to rostromedial and penetrates the thoracolumbar fascia through the orifice just before crossing the iliac crest. We then cut the thoracolumbar fascia until we reached a point where the SCN was free of kinks. Using microscissors, we opened the orifice in the distal to rostral direction along the SCN (Fig. 2). Although intraoperative manual direct compression of the SCN failed to elicit radiating pain, the presence of a local trigger point on the iliac crest suggested entrapment of other branches of the SCN. Immediately after surgery, patients reported improvement of their LBP.

\section{Evaluation of Treatment Outcomes}

Treatment outcomes were assessed by comparing the numerical rating scale (NRS) for LBP, the Japanese Orthopaedic Association (JOA) scale, and the Roland-Morris Disability Questionnaire (RDQ) scores, which were recorded before surgery and at the last follow-up. For statistical analysis, data were subjected to the paired Student t- 
TABLE 1. Characteristics of patients with SCNEN and intermittent LBP

\begin{tabular}{cccccccccc}
\hline & Age $(\mathrm{yrs})$, & & IMC Distance & Symptom & & & \multicolumn{3}{c}{ Preop/Postop Score } \\
\cline { 7 - 10 } Case No. & Sex & Affected Side & $(\mathrm{m})$ & Duration (mos) & Side of Op & FU (mos) & RDQ & JOA & NRS for LBP \\
\hline 1 & $77, \mathrm{M}$ & Lt & 30 & 24 & Lt & 16 & $6 / 0$ & $18 / 28$ \\
\hline 2 & $43, \mathrm{M}$ & Bilat & 300 & 156 & Rt & 22 & $5 / 0$ & $23 / 28$ & $7 / 1$ \\
\hline 3 & $74, \mathrm{~F}$ & Bilat & 100 & 60 & Bilat & 19 & $19 / 0$ & $16 / 26$ & $9 / 1$ \\
\hline 4 & $66, \mathrm{M}$ & Lt & 50 & 3 & Lt & 16 & $16 / 0$ & $10 / 24$ & $9 / 1$ \\
\hline 5 & $70, \mathrm{~F}$ & Bilat & 200 & 14 & Lt & 15 & $13 / 0$ & $17 / 28$ \\
\hline
\end{tabular}

$\mathrm{FU}=$ follow-up; IMC = intermittent claudication.

test using StatMate III software (ATMS Co., Ltd.). Differences of $\mathrm{p}<0.05$ were considered statistically significant.

\section{Results}

\section{Patient Characteristics}

None of the 5 enrolled patients suffered LBP at rest; LBP involving the iliac crest and buttocks was induced by standing or walking. They reported mild numbness and radiating pain in the SCN area upon compression of the trigger point corresponding to the nerve compression zone.

The walking distance that elicited intermittent LBP averaged $136 \mathrm{~m}$ (range 30-300 m). The preoperative NRS, JOA, and RDQ scores averaged 7.8 points (range 6-9), 16.8 points (range $10-23$ ), and 11.8 points (range 5-19), respectively.

First, we performed SCN block to treat patients' SCNEN; in 2 patients ( 2 sites) this produced improvement. The senior author (T.I.) operated on 6 sites in 5 patients. Surgical treatment led to significant improvement of patients' LBP and their postoperative NRS, JOA, and RDQ scores were 0.6 points (range $0-1$ ), 26.8 points (range 24-28), and 0 , respectively $(\mathrm{p}<0.05)$.

\section{Illustrative Case}

A 66-year-old man (Case 4) had a 3-month history of LBP, conservatively treated elsewhere with medications including NSAIDs. His symptoms gradually worsened, and he had difficulty sitting down and rising from a sitting position. At the time of admission, he reported LBP in the left lateral iliac crest region with radiation to the left lateral buttock, which interfered with his daily activities. Although his LBP did not manifest when he sat for prolonged periods, it worsened with gait in the lumbar-extension posture and became intolerable when he walked bent over for more than $50 \mathrm{~m}$. Whereas backward bending was painful and limited, forward bending was not. Straight leg raising, Freiberg, Pace, and Newton tests were negative. Palpation over the SIJ, spinous processes, and interspinous ligaments was unremarkable. Trigger points eliciting severe pain were located at the left lateral iliac crest of the superior rim. Tactile stimulation elicited pain radiating from the low back down to the posterior thigh (Fig. 1). Radiological examinations revealed only mild LSS (Fig. 3). Although SCN block performed 4 times dramatically reduced his pain, it returned after only a few days. Consequently, we decided to perform surgery for SCNEN.

Postoperatively, his LBP was improved and lumbar extension was possible immediately after surgery. His slouching gait posture improved, he was able to walk for longer distances, and sitting down and rising from the sitting position became easy. His NRS fell from 9 to $1 \mathrm{im}-$ mediately after the operation and remained at that level 16 months after surgery. His JOA and RDQ scores were also improved at the last follow-up (JOA score, from 10/29 to $24 / 29$; RDQ score, from $16 / 24$ to $0 / 24$ ), and he suffered no recurrence.

\section{Discussion}

The SCN develops from the rami laterales of the posterior branch of the spinal nerve and penetrates the thora-
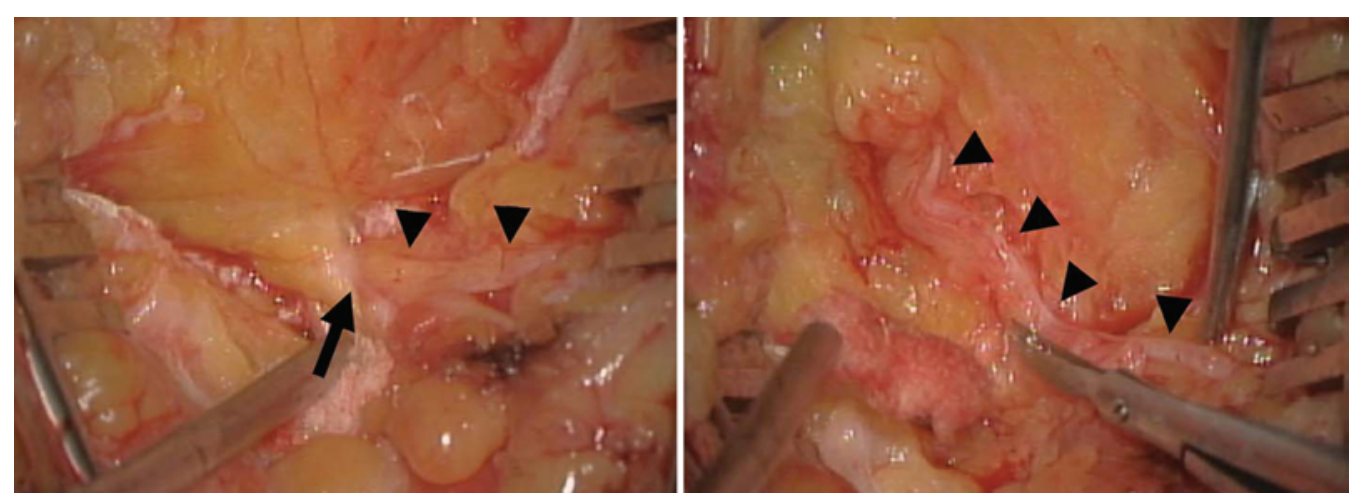

FIG. 2. Photographs showing the surgical field of the SCN entrapment (arrowheads) around the orifice (arrow) of the thoracolumbar fascia (left). Using microscissors under the microscope, we opened the orifice in the distal to rostral direction along the SCN to release the entrapped SCN (right). Figure is available in color online only. 


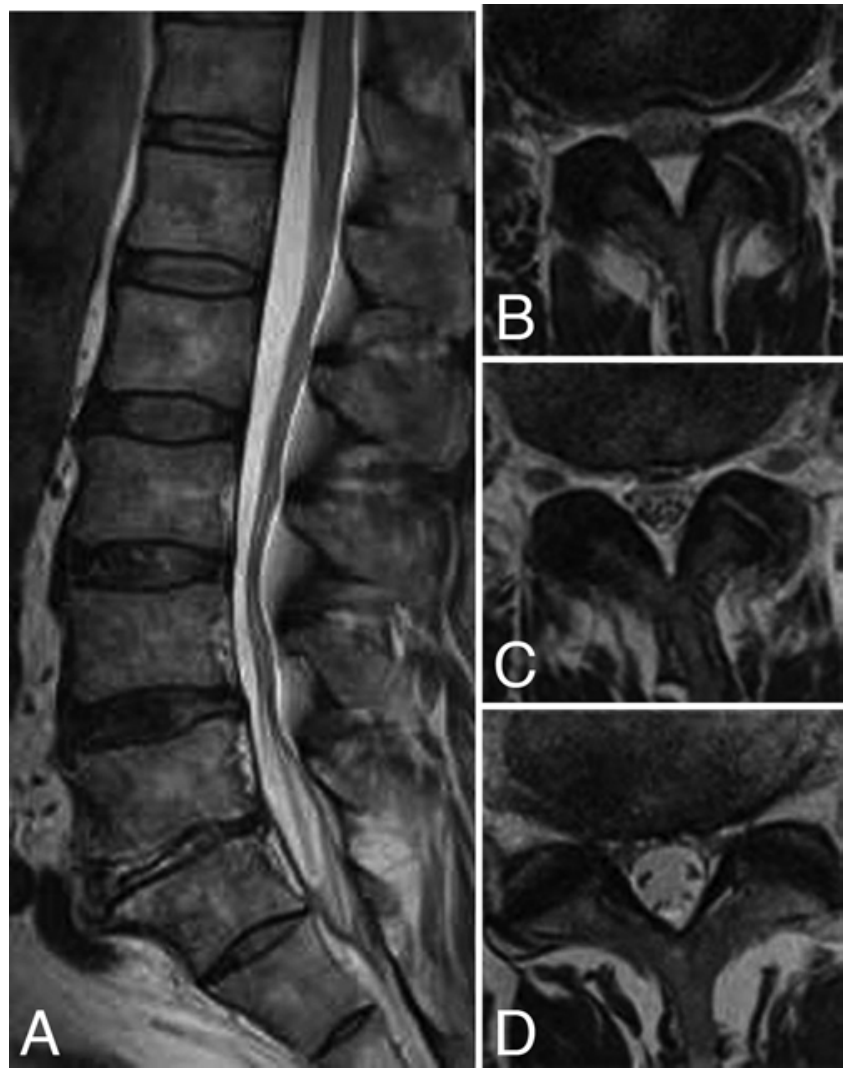

FIG. 3. Sagittal (A) and axial (L3-4 [B], L4-5 [C], and L5-S1 [D]) T2weighted MR images revealing mild lumbar spinal canal stenosis.

columbar fascia to occupy the upper buttock region over the iliac crest. ${ }^{9,11} \mathrm{SCN}$ branches extend from a site of perforation on the fascia to innervate the buttocks cutaneously. ${ }^{1,9}$ SCN entrapment at the osteofibrous orifice where it penetrates the thoracolumbar fascia produces LBP.9, ${ }^{91,13}$ In fact, $1.6 \%-12.0 \%{ }^{8,13}$ of LBP has been reported to be due to SCNEN. Surgical release at the point where the SCN exits through the osteofibrous orifice is effective. .,13-15,18 $^{-12}$

Low-back pain due to SCN entrapment is induced and exacerbated by movements such as rising, sitting, rolling over, crouching, ${ }^{1}$ lateral bending and rotating, ${ }^{1,18}$ and by prolonged sitting, ${ }^{2}$ standing, or walking. ${ }^{14}$ However, its etiology remains poorly understood.

Factors involved in the manifestation of LBP are ligaments, facet joints, the vertebral periosteum, paravertebral musculature and fascia, and spinal nerve roots. ${ }^{4}$ As many as $85 \%$ of patients with isolated LBP cannot be given a precise pathoanatomical diagnosis. ${ }^{4}$ It is difficult to base a diagnosis on radiological findings alone. To understand its origin, clinical observations and an understanding of its specific features are necessary.

Intermittent claudication, LBP associated with spinal stenosis on imaging studies, and symptom abatement with spine flexion are suggestive of LSS. ${ }^{4-6}$ LBP due to SCNEN is exacerbated by lumbar extension, improved with flexion posture, $, 14,18$ and both forward and backward bending are painful and limited in range. ${ }^{1}$ LBP tends to be misdiagnosed as lumbar spine disorder, and its clinical features and etiology remain poorly understood..$^{13}$ In our search of the literature, we found no studies on the relationship between intermittent LBP and SCNEN. Patients in the present study suffered LBP that was triggered by prolonged walking and standing. The trigger point pain localized to the iliac crest with radiation to the ipsilateral buttock; this is a clinical finding for SCNEN.,14 SCN block resulted in dramatic pain reduction, and we diagnosed SCNEN based on our criteria. ${ }^{14}$ Patients were incapable of prolonged walking or standing, and their LBP was affected by their lumbar posture-it worsened upon backward bending (lumbar extension) and was alleviated by forward bending. To our knowledge, ours is the first report suggesting that SCNEN induces intermittent LBP.

Gradual pain exacerbation in the compression posture is a symptom of entrapment neuropathy. The affected nerve becomes irritated and ischemic due to prolonged dynamic compression; these phenomena are confirmed by the Phalen test in patients with carpal tunnel syndrome. ${ }^{10}$ In patients in the present study, LBP due to SCNEN gradually increased in the lumbar extension posture, and they walked in flexion posture to avoid lumbar extension. In the lumbar extension posture, their numbness and radiating pain in the SCN area increased transiently. During surgery, we observed stronger compression of the SCN at the orifice of the thoracolumbar fascia. After release of the SCN entrapment by opening the orifice, their symptoms were no longer induced by the lumbar extension posture. These findings indicate that lumbar extension may elicit the symptoms of SCN entrapment due to attachment of the SCN to the thoracolumbar fascia. Findings upon trigger point compression may be useful for the diagnosis of SCNEN for which etiology may involve SCN traction due to changes in the orifice around the SCN. Also, manual pressure placed on the trigger point may elicit pain due to SCN traction.

Our study has some limitations. The number of patients was small, and the postoperative follow-up period was relatively short (mean 17.6 months). Although SCNEN cannot be diagnosed based on radiological findings alone, our patients manifested only mild LSS on radiological studies. We did not consider these findings to account for their intermittent claudication and LBP. To confirm that intermittent LBP is due to SCNEN, we are planning to perform large cohort studies. In the meantime, we suggest that SCNEN be considered in the differential diagnosis of intermittent LBP.

\section{Conclusions}

We report outcomes for 5 patients with intermittent LBP due to SCNEN. We present clinical and surgical findings showing that their LBP was exacerbated by lumbar extension. Our patients obtained symptom relief by $\mathrm{SCN}$ block or surgical release of the SCN entrapment. Based on these observations, we suggest that SCNEN be considered as a causal factor in patients with LBP elicited by walking.

\section{References}

1. Akbas M, Yegin A, Karsli B: Superior cluneal nerve entrapment eight years after decubitus surgery. Pain Pract 5:364366,2005 
2. Aly TA, Tanaka Y, Aizawa T, Ozawa H, Kokubun S: Medial superior cluneal nerve entrapment neuropathy in teenagers: a report of two cases. Tohoku J Exp Med 197:229-231, 2002

3. Berthelot JM, Delecrin J, Maugars Y, Caillon F, Prost A: A potentially underrecognized and treatable cause of chronic back pain: entrapment neuropathy of the cluneal nerves. J Rheumatol 23:2179-2181, 1996

4. Deyo RA, Weinstein JN: Low back pain. N Engl J Med 344:363-370, 2001

5. Hilibrand AS, Rand N: Degenerative lumbar stenosis: diagnosis and management. J Am Acad Orthop Surg 7:239249, 1999

6. Katz JN, Dalgas M, Stucki G, Katz NP, Bayley J, Fossel AH, et al: Degenerative lumbar spinal stenosis. Diagnostic value of the history and physical examination. Arthritis Rheum 38:1236-1241, 1995

7. Kim K, Isu T, Chiba Y, Morimoto D, Ohtsubo S, Kusano M, et al: The usefulness of ICG video angiography in the surgical treatment of superior cluneal nerve entrapment neuropathy: technical note. J Neurosurg Spine 19:624-628, 2013

8. Kuniya H, Aota Y, Nakamura N, Kawai T, Tanabe H, Saito T: [Low back pain patients with suspected entrapment of the superior cluneal nerve.] J Spine Res 2:1032-1035, 2011 (Jpn)

9. Kuniya H, Aota Y, Saito T, Kamiya Y, Funakoshi K, Terayama $\mathrm{H}$, et al: Anatomical study of superior cluneal nerve entrapment. J Neurosurg Spine 19:76-80, 2013

10. Kuschner SH, Ebramzadeh E, Johnson D, Brien WW, Sherman R: Tinel's sign and Phalen's test in carpal tunnel syndrome. Orthopedics 15:1297-1302, 1992

11. Lu J, Ebraheim NA, Huntoon M, Heck BE, Yeasting RA: Anatomic considerations of superior cluneal nerve at posterior iliac crest region. Clin Orthop Relat Res (347):224-228, 1998

12. Mahli A, Coskun D, Altun NS, Simsek A, Ocal E, Kostekci M: Alcohol neurolysis for persistent pain caused by superior cluneal nerves injury after iliac crest bone graft harvesting in orthopedic surgery: report of four cases and review of the literature. Spine (Phila Pa 1976) 27:E478-E481, 2002

13. Maigne JY, Doursounian L: Entrapment neuropathy of the medial superior cluneal nerve. Nineteen cases surgically treated, with a minimum of 2 years' follow-up. Spine (Phila Pa 1976) 22:1156-1159, 1997

14. Morimoto D, Isu T, Kim K, Imai T, Yamazaki K, Matsumoto $\mathrm{R}$, et al: Surgical treatment of superior cluneal nerve entrapment neuropathy. J Neurosurg Spine 19:71-75, 2013
15. Speed S, Sims K, Weinrauch P: Entrapment of the medial branch of the superior cluneal nerve. A previously unrecognized cause of lower back pain in cricket fast bowlers. J Med Cases 2:101-103, 2011

16. Strong EK, Davila JC: The cluneal nerve syndrome; a distinct type of low back pain. Ind Med Surg 26:417-429, 1957

17. Takahashi I, Kikuchi S, Sato K, Iwabuchi M: Effects of the mechanical load on forward bending motion of the trunk: comparison between patients with motion-induced intermittent low back pain and healthy subjects. Spine (Phila Pa 1976) 32:E73-E78, 2007

18. Talu GK, Ozyalçin S, Talu U: Superior cluneal nerve entrapment. Reg Anesth Pain Med 25:648-650, 2000

19. Tubbs RS, Levin MR, Loukas M, Potts EA, Cohen-Gadol AA: Anatomy and landmarks for the superior and middle cluneal nerves: application to posterior iliac crest harvest and entrapment syndromes. J Neurosurg Spine 13:356-359, 2010

20. Zhong J: Acupuncture treatment in 96 cases of superior cluneal nerve injury. J Tradit Chin Med 11:259-260, 1991

\section{Disclosure}

The authors report no conflict of interest concerning the materials or methods used in this study or the findings specified in this paper.

\section{Author Contributions}

Conception and design: Chiba, Isu, Kim. Acquisition of data: Chiba, Isu, Kim, Iwamoto. Analysis and interpretation of data: Chiba, Isu, Kim. Drafting the article: Chiba, Kim. Critically revising the article: Chiba, Kim, Morimoto, Yamazaki, Hokari, Isobe, Kusano. Reviewed submitted version of manuscript: all authors. Approved the final version of the manuscript on behalf of all authors: Chiba. Statistical analysis: Chiba, Kim. Administrative/technical/material support: Chiba, Isu, Iwamoto, Isobe, Kusano. Study supervision: Chiba, Isu, Kim, Isobe, Kusano.

\section{Correspondence}

Yasuhiro Chiba, Department of Neurosurgery, Kushiro Rosai Hospital, 13-23, Nakazono-cho, Kushiro, Hokkaido 085-8533, Japan.email: chibay511@hotmail.com. 